\title{
The hybrid algorithm for chronic total occlusions
}

\author{
Carlo Zivelonghi, Simone Budassi, Pierfrancesco Agostoni \\ Hartcentrum, Ziekenhuis Netwerk Antwerpen (ZNA) Middelheim, Antwerp 2020, Belgium. \\ Correspondence to: Dr. Pierfrancesco Agostoni, Hartcentrum, Ziekenhuis Netwerk Antwerpen (ZNA) Middelheim, Antwerp \\ 2020, Belgium. Email: agostonipf@gmail.com
}

How to cite this article: Zivelonghi C, Budassi S, Agostoni P. The hybrid algorithm for chronic total occlusions. Vesse/ Plus 2019;3:30. http://dx.doi.org/10.20517/2574-1209.2019.06

Received: 4 Feb 2019 First Decision: 21 Jun 2019 Revised: 22 Jun 2019 Accepted: 31 Jul 2019 Published: 14 Aug 2019

Science Editor: Mario F. L. Gaudino Copy Editor: Jia-Jia Meng Production Editor: Jing Yu

\begin{abstract}
In the last years procedural success rate of chronic total occlusions (CTOs) percutaneous coronary intervention has improved primarily for two reasons: the evolution in materials and the new techniques and skills acquired by dedicated CTOs operators. In the last decade a lot of complex and advanced CTO techniques have been introduced. The hybrid algorithm allows to standardize the experience shared by CTO operators. The aim of the algorithm is to help the operators to choose the best strategy for the single case, in order to improve procedural success rate, to fasten the procedure, shortening failure modes, and to reduce $\mathrm{X}$ ray exposure and contrast load. The aim of our review is to highlight the most recent scientific evidence about the use of the hybrid algorithm for the treatment of CTO.
\end{abstract}

Keywords: Chronic total occlusion, percutaneous coronary intervention, interventional cardiology, coronary artery disease, hybrid algorithm

\section{INTRODUCTION}

In the last years the success rate of chronic total occlusions (CTOs) percutaneous coronary interventions (PCI) has rapidly improved, not only because of the evolution in materials (e.g., microcatheters, dedicated guidewires) but also thanks to the development of dedicated programs and shared strategies for operators approaching these very complex procedures. In addition, a major role has been played by the introduction of new complex techniques, including retrograde approaches and sub-intimal strategies. In details, dissection/re-entry techniques consist in gaining the sub-intimal space before/inside the CTO and re-entering in the true-lumen of the native vessel once the occlusion segment is overtaken. This can 


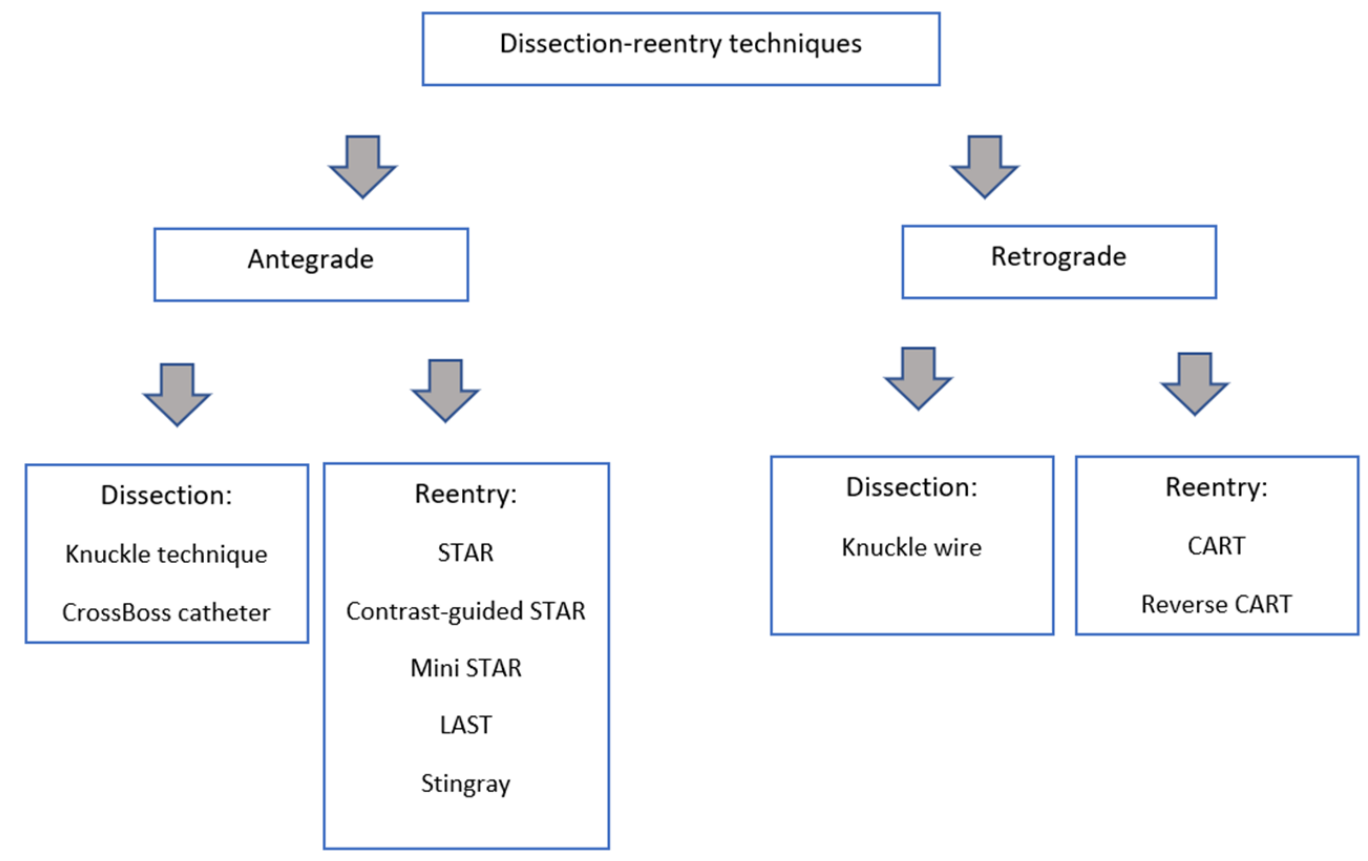

Figure 1. Flowchart showing the possible dissection/re-entry techniques. CART: controlled antegrade and retrograde subintimal tracking; LAST: limited antegrade subintimal tracking; STAR: subintimal track and reentry

be performed both via retrograde or antegrade approaches [Figure 1]. One of the first techniques was introduced by Colombo et al. ${ }^{[1]}$ namely the subintimal track and reentry (STAR) technique (this technique was first developed for peripheral interventions). To obtain the "dissection and re-entry" Colombo used a polymer jacket looped guidewire ("knuckle wire") such as the Fielder-XT or the Pilot 200, that entered easily in the sub-intimal space before the occlusion (because of its hydrophilic properties) and could manage to spontaneously re-enter in the true lumen when approaching a bifurcation in a more distal segment of the vessel. Since its first description in 2005, this technique has experienced many evolutions according to different operators. In 2008, Carlino et al. ${ }^{[2]}$ reported for the first time its subintimal technique, performed by placing a microcatheter in the subintimal space, and injecting contrast to visualize the subintimal course and thus facilitating driving of the guidewire. This technique is known as contrast-guided STAR technique. In addition, the limited antegrade subintimal tracking (LAST) technique has been introduced by Lombardi ${ }^{[3]}$ in 2009: this consists in the progression of a guidewire in the subintimal space, performing a re-entry as proximal as possible after the occlusion. The guidewire must be previously knuckled to create a loop with a very acute angle, to facilitate the re-entry. With the introduction of the CrossBoss and the Stingray Catheters (Boston Scientific) since 2012 the success rate of the CTO dissection re-entry procedure has been improved. In the mini-STAR technique introduced by Galassi et $a l .{ }^{[4]}$ since 2012 a microcatheter (generally the Finecross, Terumo, Tokyo Japan) is advanced up to the proximal cap of the CTO using a spring soft guidewire that must be exchanged with a Fielder wire that addressed the true lumen.

Focusing now on retrograde approach, the retrograde dissection can be obtained with a knuckle guidewire, the re-entry can be achieved with two different techniques. The first one is the controlled antegrade and retrograde subintimal tracking (CART) technique, introduced by Surmely et al. ${ }^{[5]}$ in 2006. As a first step, the operator should place the retrograde guidewire into the subintimal space. A balloon is advanced through the retrograde guidewire and inflated in the subintimal space in order to track the advancing of the antegrade guidewire into the distal vessel. The second (and currently most common) is the reverse CART technique, which is conceptually similar to the previous technique but inverted. The balloon is 


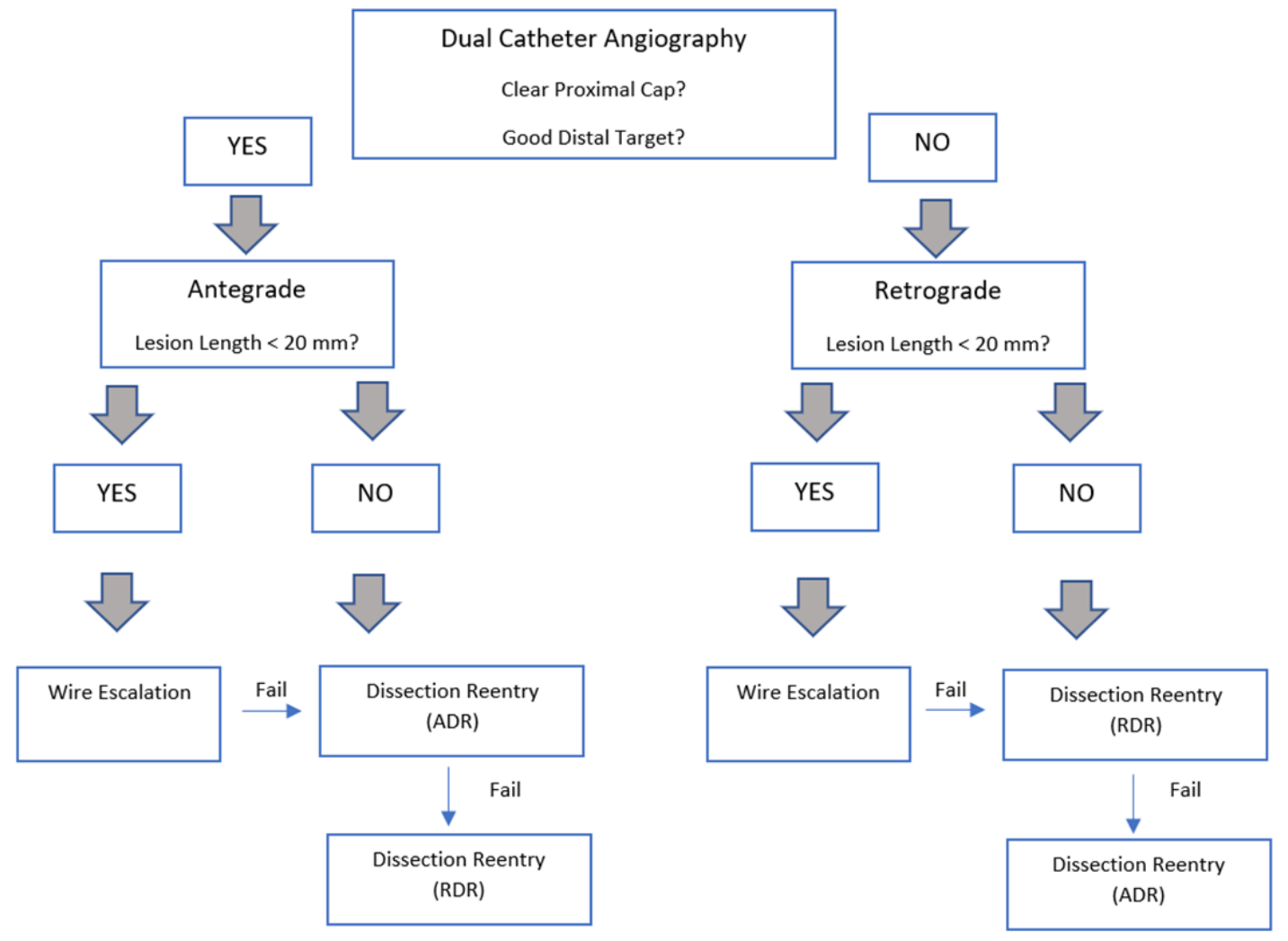

Figure 2. Illustrative representation of the hybrid algorithm. It is important to highlight the possibility to switch from one technique to the other if the previous technique is in failure mode. ADR: antegrade dissection and re-entry; RDR: retrograde dissection and re-entry

positioned in the subintimal space thorough the antegrade guidewire. Then the balloon is inflated to expand the subintimal space and to facilitate the transition of a retrograde guidewire from the subintimal space connected with the proximal true lumen.

The hybrid algorithm [Figure 2] was first described by Brilakis ${ }^{[6]}$ and colleagues in 2012, in contrast to the Japanese School that traditionally favors true lumen-to-true lumen techniques. Given this variety of new techniques and increasingly dedicated materials, the aim of the hybrid algorithm was to optimize not only success but also time and procedural steps by suggesting an easy switch among techniques to select the "right technique for the right CTO". Theoretical additional advantage of such approach is also that of allowing reduction in exposure times for the operator and the patient. The hybrid approach indeed includes all available CTO techniques. It starts mandatorily with dual-injection angiography. In order to select the best strategies, the operator focuses on 4 main anatomic characteristics of the lesion: (1) proximal cap ambiguity; (2) distal target vessel; (3) interventional collateral; and (4) lesion length ${ }^{[7]}$. After careful angiographic evaluation, bailout and consecutive strategies are determined upfront. It is recommended to change strategy after not more than 5-10 min without any progression.

Despite the excellent results of this algorithm in the USA and Europe, in the Asian regions this is rarely applied. The Asian Pacific CTO club has therefore introduced a new type of algorithm since 2017. There are three characteristics that guide the operator in choosing the primary strategy and if it has to be anterograde or retrograde. The three features are: proximal cap ambiguity, the quality of the vessel distally and if there are good caliber collateral branches. Unlike the Hybrid approach, the length of the lesion alone is not enough to determine whether a wire escalation or re-entry dissection technique should be more advisable ${ }^{[8]}$. 
The aim of our review is to highlight the most recent scientific evidence about the use of hybrid algorithm for the treatment of CTO.

\section{OUTCOMES OF THE HYBRID ALGORITHM}

\section{Multicenter US registry}

An important Multicenter registry from the USA examined the procedural technique and outcomes of 1036 consecutive CTO PCI using the hybrid algorithm between 2012 and 2015 in 11 US centers. Technical success was achieved in $91 \%$ and a major procedural complication occurred in $1.7 \%$ of the cases. The initial crossing strategy was successful in $58 \%$ of the lesions, whereas $39 \%$ required an additional approach. In this registry, the application of the hybrid approach resulted in high procedural success and low complication rate, supporting its expanding use in $\mathrm{CTO} \mathrm{PCI}^{[9]}$. It is important to notice that the physicians participating in this registry were extremely experienced CTO operators with already years of expertise in CTO techniques, thus the external validity of the results of this registry in a group of physicians with less CTO expertise is debatable.

\section{The RECHARGE registry}

A solid source of data was also provided by the REgistry of Crossboss and Hybrid procedures in FrAnce, the NetheRlands, BelGium and UnitEd Kingdom (RECHARGE $)^{[10]}$, which described the procedural and clinical outcome of the adoption of the hybrid algorithm in Europe in a group of physicians with definitely less expertise (during the registry) than the American doctors involved in the Multicenter US registry. In this prospective, multicenter registry, patients treated electively for CTO PCI were prospectively included from 17 centers between January 2014 and October 2015. A total of 1253 CTO PCI were performed. The average J-CTO score was $2 \pm 1$ and was higher in the failure group ( $2.6 \pm 0.6 v s .1 .9 \pm 1, P<0.001)$. Overall procedural success was $86 \%$ and major in-hospital complications rate was $2.6 \%$. The primary strategy was successful in $60 \%$ of cases; a switch to a second-line strategy (as suggested by the principle of the algorithm) occurred in $34 \%$ of cases, resulting highly successful (74\%). Median procedural time was 90 min (IQR 60$120 \mathrm{~min}$ ) and median fluoroscopy time was $35 \mathrm{~min}$ (IQR 21-55 min), median contrast volume was $250 \mathrm{~mL}$ (IQR 180-340 mL). High technical success rates combined with a low event rate supported further use of this algorithm. Four techniques were mainly applied: antegrade wire excalation (AWE) was the primary strategy in $77 \%$ of patients, followed by a retrograde technique in $17 \%$ and antegrade dissection reentry in $7 \%$. The primary strategy was successful in $91 \%$ of easy procedures (J-CTO score 0 ), $80 \%$ of intermediate (J-CTO score 1), $62 \%$ of difficult (J-CTO score 2) and $43 \%$ of very difficult lesions (J-CTO > 2). Second, third or more bailout crossing strategies were used in $34 \%$ of cases and were successful in $74 \%$ of the procedures, leading to an overall technical success rate of $86 \%$.

Of note, a sub-analysis of the RECHARGE study was focused on investigating the feasibility and efficacy of the radial approach in CTO PCI. The authors analyzed the 1253 patients undergoing CTO PCI according to the hybrid algorithm and divided the population into two groups: a fully trans-radial approach (fTRA, single o bi-radial accesses) vs. transfemoral approach (TFA, defined as single femoral, bi-femoral or combined femoral/radial). A fTRA was applied in 306 (24\%) cases, while 947 patients (76\%) were treated in the TFA group. Technical success was achieved in 259 of 306 patients (85\%) in the fTRA group and 816 of 947 patients (86\%) in the TFA group, and these similar success rates was also confirmed after propensity score matching and stratification for J-CTO score. This was one of the first studies supporting the efficacy of the radial approach as a valid alternative to the conventional trans-femoral one in CTO PCI ${ }^{[11]}$.

\section{The four angiographic characteristics of the hybrid algorithm}

1. Proximal cap ambiguity: this refers to the possibility to clearly identify and define the entry point of the CTO lesion and to engage it. 
2. Lesion length: lesions are divided in less versus more than $20 \mathrm{~mm}$. Lesions $\geq 20 \mathrm{~mm}$ have a longer procedure time and lower success rate using standard wire escalation techniques ${ }^{[12]}$. In this case the best primary approach may be dissection reentry techniques.

3. Target coronary vessel at the distal cap: this refers to the size of the lumen after the distal CTO cap, distally to the lesion itself, and the eventual presence of significantly visible side branches at the distal cap level; this characteristic has also an impact on the possibility to perform dissection/re-entry techniques.

4. Size and suitability of collateral circulation for retrograde techniques (so called "interventional collaterals"): optimal collateral vessels can be easily accessed with wires and microcatheters, are large enough to allow the passage of these devices, have minimal tortuosity and are not the only source of flow ${ }^{[6]}$.

\section{DEVICES TO SUPPORT PCI}

\section{Wires}

1. A hydrophilic and/or polymer-jacket 0.014 " guidewire, low-gram force with tapered tip for outweigh microchannel or soft tissue, for collateral channel passage and for knuckle techniques (examples: Fielder XT, Fielder XT-A, Fielder XT-R, all Asahi Intecc, Nagoya Japan).

2. A non-tapered, polymer-jacket hydrophilic 0.014 " guidewire for collateral channel crossing in retrograde approach. (examples: Fielder FC, Suoh 03, Sion black, all Asahi Intecc, and Pilot 50, Abbott Vascular Santa Clara, California).

3. A moderately high gram force (from 4 to $6 \mathrm{~g}$ ) polymer-jacket non-tapered guidewire for complex lesion crossing, knuckle technique and dissection re-entry, such as Pilot 200 (Abbott vascular) guidewire.

4. A high-gram-force 0.014 " guidewire with a tapered non-jacketed tip for penetration techniques, cap puncture, complex lesions crossing and lumen re-entry techniques, for example Miracle 12, Confianza Pro 12 wire (Asahi Intecc) or Hornet 14 (Boston Scientific).

\section{Microcatheters}

1. Corsair (Asahi Intecc): it's a $2.7 \mathrm{~F}$ microcatheter. It is originally designed for septal collateral crossing. The length for this use is $150 \mathrm{~cm}$. Thanks to the screw like structure of the distal part, which uses 2 thick stainless-steel wires and 8 thin stainless-steel wires, it ensures very high crossability into tortuous vessels during retrograde approach. It reinforces the torque transmission to the guide wire and creates better backup for penetration of harder lesions. An antegrade version to support antegrade techniques (wire support and exchange) has been developed with a length of $135 \mathrm{~cm}$. Both the $5 \mathrm{~mm}$ tapered soft tip with Tungsten powder mix and the reinforced tapered shaft after the screw like structure, are coated with a Hydrophilic Polymer, which provides lubricity and enhances maneuverability. It also allows super selective injection of contrast.

2. Caravel (Ashai): the lengths are $135 \mathrm{~cm}$ (for antegrade approach) and $150 \mathrm{~cm}$ (for retrograde approach), the tip entry profile is $0.48 \mathrm{~mm}$ and the microcatheter is coated with hydrophilic coating. It is compatible with 0.014 " wire. It has good crossing profile and trackability and the low-profile shaft facilitates the crossing of microchannels.

3. Turnpike (Teleflex): it's a "family" of microcatheters containing a robust multi-layer shaft that provides enhanced flexibility, torque and tracking over a 0.014" guidewire. There are: the Turnpike (antegrade version $135 \mathrm{~cm}$, retrograde $150 \mathrm{~cm}$ ), the Tunpike Low Profile (LP, antegrade version $135 \mathrm{~cm}$, retrograde $150 \mathrm{~cm}$ ), the Turnpike Spiral (only antegrade $135 \mathrm{~cm}$ ) and the Turnpike Gold (only antegrade $135 \mathrm{~cm}$ ). 
4. Small outer diameter over the wire (OWT) microcatheters like Finecross (Terumo-corporation), Quick Cross (Spectranetics corporation, Colorado) or Supercross (Teleflex) for wire support and exchange. Also of these catheters an antegrade and a retrograde version exists.

5. Small OWT balloons for support and exchange are in general not recommended in contemporary CTO PCI.

6. Tornus microcatheter (Asahi, Intecc): Tornus has a solid metal body, while the outer surface is hydrophobic coated. It is a penetration microcatheter that allows to expand the lumen of calcified vessels, providing easy access to other medical devices and improving the treatment of tight lesions. It allows to cross CTO through helical movements.

7. CrossBoss microcatheter (Boston Scientific): this is a metal OTW microcatheter with a rounded "oliveshaped" tip. Once inserted in the body of the CTO lesion, it is rotated rapidly in either direction to facilitate advancement through a CTO without the wire in the lead. It is a key device for controlled antegrade subintimal dissection/re-entry techniques ${ }^{[13]}$. It creates a controlled blunt dissection of the segment tracked. Due to its shape, the chance to perforate is limited. The device tends to maintain a subintimal position during advancement. It is used only during antegrade procedures.

8. Stingray balloon and Stingray guidewire systems (Boston Scientific): it is a 1-mm flat dual-parallel balloon with 3 exits ports connected to the same guidewire lumen ${ }^{[13]}$. It is a device for reentry purposes distal to the CTO segment (thus to be used only in antegrade procedures). The distal exit port is used to place the balloon in position. When the balloon is inflated it generates a flat surface (due to the 2 parallel balloons) that accommodates itself in the subintimal space around the intima. Once inflated, the other two exit ports, which are both just before the distal one, are $180^{\circ}$ opposed, so one is oriented to the adventitia and the other to the lumen. It is used for true lumen re-entry ${ }^{[6]}$.

To manage complications like coronary perforation and cardiac tamponade the Cath lab must be equipped with covered stents, embolization coils, pericardiocentesis kit and the operators should be trained for the treatment of these dangerous complications.

\section{TECHNIQUES}

\section{Access}

The operator should use access routes which he/she is more familiar with ${ }^{[14]}$. On one hand the femoral access allows the use of larger guiding catheters improving the support, on the other hand the radial approach improves patient comfort and reduces complications ${ }^{[15]}$. Both are acceptable. In the sub-analysis from the RECHARGE trial, the use of a fTRA resulted feasible in the whole spectrum of difficulties for CTO revascularization ${ }^{[1]}$. In these cases, more supportive guiding catheters are preferred to allow enough penetrative power and overcome the most resilient proximal cap. Guiding catheter size is usually limited to 6-7 French (occasionally $7 F$ ) from the radial approach, compared with standard 8 French used in transfemoral CTO $\mathrm{PCI}^{[16]}$. All the operators but especially radial operators, should be familiar with all the techniques that can improve the support like balloon anchoring, mother and child techniques and guiding extension devices ${ }^{[17]}$. The balloon anchoring is achieved by inflating a balloon into a small branch of the target vessel proximal to the CTO segment to obtain support to overcome the lesion. The mother and child technique provides to insert a smaller French catheter into a larger catheter to improve support to the guidewire. Nowadays guiding catheter extension devices such as Guideliner (Teleflex) and Guidezilla (Boston Scientific) are available and better choice than mother and child guiding catheters. It is recommended to start the procedure with ipsilateral and contralateral injection for a proper diagnostic 
angiography, to be able to know adequately the anatomy and to choose properly the best primary approach and the eventual following approaches. We suggest unfractionated heparin as the best form of anticoagulation during CTO PCI, with an activated clotting time more than $350 \mathrm{~s}$. Heparine is considered the safest anticoagulation method because in case of complications it can be rapidly reversed with protamine, if needed.

\section{Antegrade wire escalation}

It is the sequential use of guidewires with different characteristics from the proximal to the distal part of the lesion in order to try and remain intraluminal during the whole trajectory and to achieve directly the distal true lumen after the CTO. It is the most commonly used primary strategy in CTO PCI (around three quarter of the cases) ${ }^{[10]}$. Following the hybrid algorithm, you can choose AWE as primary strategy in case of: (1) an unambiguous proximal cap; (2) < $20 \mathrm{~mm}$ occlusion length; and (3) good distal target vessel. Soft tapered polymeric wires are the initial choice. These wires can cross the occlusion through small invisible channels or by crossing the softest part of the lesion. After trying for a few minutes without progression, it is better to rapidly step up to stiff spring coil tapered wires to overcome hard and fibrotic/calcified segments, and then change again with a soft wire to complete the crossing of the $\mathrm{CTO}^{[7]}$. Penetration force and maneuverability of the wire should always be supported by a microcatheter ${ }^{[18]}$. The CTO crossing wires should be exchanged once they access the distal true lumen, with safer wires, to prevent distal small vessel perforation. A possible AWE technique can be applied when the first wire is constantly directing into the false lumen: the parallel wire technique. The first wire is left in the false lumen and a second wire is passed parallel: by doing so, the first wire keeps the dissection channel closed and acts as marker for advancing the second wire ${ }^{[19]}$.

\section{Anterograde dissection re-entry techniques}

Antegrade dissection occurs when a guidewire or a microcatheter is advanced within the subintimal space. This can be obtained with the CrossBoss catheter or a knuckle wire technique ${ }^{[4,6]}$. In the latter approach, which is also the first step of the STAR technique, a 360 degrees looped polymer jacketed wire (the knuckle wire) is advanced in the direction of the distal cap of the CTO. It is important not to rotate the knuckle wire to avoid entanglement. This approach is known to be much safer than advancing stiffer guidewires without knuckle, as the knuckle tracks the vessel anatomy in the subintimal space without perforating the vessel. The CrossBoss catheter has the advantage of creating a smaller sub adventitial space than the knuckle wire, further it can facilitate the use of the Stingray system for final re-entry in the true lumen, as it created a larger subintimal channel. After subintimal crossing there are two primary method to re-entry: (1) LAST Method; (2) device-based (Stingray system).

\section{The LAST Method}

After gaining the sub-intimal space, the wire is advanced after the distal cap of the CTO, and the microcatheter is positioned near the desired re-entry point. A dedicated guidewire (stiff polymer jacket or stiff tapered) is selected and directed to the distal true-lumen in order to obtain a successful re-entry ${ }^{[6]}$.

\section{Stingray-based reentry}

The Stingray balloon has a flat shape with two side-exit ports. Upon low-pressure (2-4 atm) inflation, one exit port is automatically oriented toward the true lumen and the other one toward the vessel adventitia ${ }^{[20]}$. The delivery catheter shaft is 0.014 " guidewire compatible. The Stingray guidewire is a stiff guidewire with a $20 \mathrm{~cm}$ distal radiopaque segment, a $1.5 \mathrm{~mm}, 28^{\circ}$ angle, distal bend, and a tapered tip with a $0.0035^{\prime \prime}$ distal prong. The Stingray guidewire is advanced through one of the two side ports of the Stingray balloon under fluoroscopic guidance to re-enter into the distal true lumen.

These techniques, specifically the Stingray-based approach, are less frequently adopted, and were often considered as the last chance in the beginning. Indeed in the RECHARGE registry and also in the data 
form the US registry the adoption rate was respectively $18 \%$ and $26 \%^{[9,10]}$. This can be attributed to the higher difficulty and lower predictability of success of this technique and the need for more expensive materials to obtain successful reentry. However, with expertise, this technique is gaining more and more space in the current strategical approach to CTO.

\section{THE RETROGRADE APPROACH}

\section{Retrograde wire crossing}

Retrograde wire crossing means CTO crossing from distal to proximal, with successful access to the true lumen in the section of the vessel upstream the lesion ${ }^{[21]}$. After successful passage, the operator brings the retrograde wire and microcatheter into the antegrade guiding catheter. The last step of this technique is to exchange the retrograde wire for a long wire to be externalized from the retrograde guiding through the microcatheter in the antegrade guiding. This externalized wire is then used as an antegrade wire from the antegrade guiding catheter ${ }^{[2]}$.

\section{Kissing wire technique}

This procedure mixes antegrade and retrograde techniques. Lesion penetration is performed antegradely. If the operator finds a microchannel or if the CTO lesion is relatively soft, the antegrade wire can be advanced; the operator can stop the wire halfway through the occlusion. The retrograde wire is then advanced aiming the tip towards the antegrade wire. Eventually, the antegrade and retrograde guide wires will meet (or "kiss"). The retrograde wire is substantially used as landmark to be followed by the antegrade wire. This technique is generally used to reduce contrast exposure for the patient and to reduce the possibility of potential ambiguity regarding the course of the vessel, thus making advancement of the antegrade wire safer. After crossing the whole lesion, the antegrade wire has to be in the true lumen of the vessel downstream the CTO lesion, the balloon catheter is advanced into the occlusion and the operator can start to dilate the lesion. The kissing wire technique is rarely performed by experienced retrograde operators, as the reverse CART technique provides a more consistent method for connecting the channels in refractory $\operatorname{cases}^{[17]}$.

\section{Knuckle technique}

As previously discussed, in the knuckle technique a dissection is created by forming a loop in the retrograde wire. Ideal wires for this purpose are soft hydrophilic wires. The use of a microcatheter is crucial for allowing increased support in advancing the knuckle wire and to allow the finalization of the technique $^{[19]}$. Conventionally used wires for the knuckle technique are the Fielder XT (generating smaller knuckle and often minor sub-intimal areas) and the Pilot 200 (generating instead bigger knuckles and wider sub-intimal areas, because of its higher stiffness; this is especially useful in performing sub-intimal techniques in the right coronary artery). In more complex scenarios, where the knuckle of the wire alone is not strong enough to allow advancement through the occlusion, the use of "microcatheter knuckle" has also been described by experienced operators. Basically, this technique consists of forming a knuckle with the wire and the microcatheter together, which is then pushed forward as a unique system in order to allow for more force to overcome extreme calcifications and resistance when performing sub-intimal tracking. The "knuckle" is a key technique in the hybrid approach armamentarium. In fact, when CTO conditions appear adverse to a common true-lumen to true-lumen technique (such as AWE or retrograde wire escalation, especially when bendings $>90^{\circ}$ and occlusion length $>20 \mathrm{~mm}$ coexist), creating an entrydissection and advancing the knuckle grants a relatively easier, faster and safer way to cross the occlusion, opening the possibilities to the various re-entry techniques.

\section{CART technique}

In the CART technique, the principle is to create a dissection upstream the CTO lesion for antegrade crossing. A guidewire with the support of a microcatheter is overlapped to a balloon catheter advanced on 
a guidewire placed in the subintimal space via the retrograde approach. The antegrade and the retrograde systems meet each other in the subintimal space Multiple dilatations of the retrograde balloon create a connection between the anterograde and the retrograde spaces so that the antegrade wire may regain the true lumen distal to the occlusion ${ }^{[19]}$. This technique is rarely used nowadays, mainly due to the complexity of tracking a balloon through the retrograde way. The reverse CART technique is currently preferred.

\section{Reverse CART technique}

Another method to connect the proximal and the distal lumen is the reverse CART technique. This method is the most commonly used. A microcatheter is placed across a collateral vessel into the subintimal space of a CTO segment, over a guidewire (via retrograde approach). An antegrade wire is advanced into the CTO segment alone or with a microcatheter or a balloon. The balloon is placed adjacent to retrograde microcatheter and inflated. The balloon angioplasty creates a connection between the two spaces. The retrograde wire can now be passed into the proximal vessel and wire externalization can be performed ${ }^{[7]}$.

\section{DISCUSSION}

According to the hybrid algorithm, a first and key point is the performance of a proper dual injection to optimally view the CTO lesion and estimate its length, the proximal cap characteristics and the quality of the distal target vessel to choose the optimal initial strategy ${ }^{[23]}$. According to Christopoulos et al ${ }^{[9]}$ the dual injection has been demonstrated crucial for achieving high success rates in CTO PCI. Indeed, the hybrid algorithm improves the success rate in CTO PCI when applied by experienced operators. In the RECHARGE ${ }^{[10]}$ the success rate was $86 \%$ with a low incidence of adverse events $(2.6 \%)$. In the multicentre US registry ${ }^{[9]}$ the success with the initial crossing strategy resulted $58 \%$, but after adoption of additional strategies a final technical success rate was $91 \%$ with a low incidence of adverse events (1.7\%). The only problem could be the interpretability of the angiographic characteristics, for example the proximal cap ambiguity is subjective, and it depends on operator experience. In the RECHARGE, despite the high degree of several negative angiographic characteristics, AWE was the most frequently used first technique (77\%). AWE was successful in $62 \%$ of the cases with low success rate in very difficult CTO PCI. This problem could be potentially bypassed using intravascular ultrasound imaging which would help better defining the proximal cap ${ }^{[10,20]}$. In the same registry the algorithm's suggestion that lesion length $\geq 20 \mathrm{~mm}$ should drive the strategy to dissection and re-entry techniques, was less followed than expected. In 50\% of cases the first strategy applied was still AWE. Two possible factors should be kept into consideration: (1) lesion length is often not clear, even if the dual injection should reduce this risk of misinterpretation; (2) operators still prefer to wire the softest tissue of the CTO with dedicated wires and new microcatheters (lower crossing profiles), while shifting to a dissection/re-entry technique is always a possible strategy in case of AWE failure. Importantly, the threshold to change strategy should be within 15 to $30 \mathrm{~min}$ of failure mode, to reduce contrast and radiation exposure. Overall the availability of subsequent strategies and the ability to shift between different ones is crucial to increase the success rate from $60 \%$ to $85 \%-90 \%{ }^{[10]}$. Indeed, as shown in the US registry, an easy switch in strategies allowed for an increase of technical success from $64 \%$ with the firstly adopted technique to a final $91 \%$. An alternative algorithm, the minimalistic hybrid approach, was described by Zivelonghi et al. ${ }^{[24]}$. The authors developed it in order to minimize the use of double access, large bore catheters and femoral approach, thus reducing patient's discomfort and procedural complications. In this sequence of steps all the techniques are included: antegrade, retrograde as well as sub-intimal and intraluminal techniques. The focus of this algorithm is to perform all the possible techniques of the hybrid algorithm minimizing the unnecessary "damage" to the patient (for example, no second access if not needed, $6 \mathrm{~F}$ guiding catheters instead of $7-8 \mathrm{~F}$ in case of "a priori" belief that the procedure can be performed with $6 \mathrm{~F}$ guiding only, default radial access in case of $6 \mathrm{~F}$ guiding). 


\section{CONCLUSION}

The application of the hybrid strategies allows to achieve extremely highly procedural success rates with limited complication incidence. Although AWE is often used as an initial technique, this approach alone has a low success rate in the most complex CTO lesions. As suggested in the hybrid algorithm, when the characteristics of the CTO-lesion appear not favorable (especially when the lesion length is more than $20 \mathrm{~mm}$ ) the direct use of a dissection re-entry technique can be considered -both antegrade or retrogradedepending on the proximal cap characteristics and the distal vessel morphology. Implementing this strategy could result in higher success rate, lower complication rate and a limitation of the radiation exposure for both operators and patients, as well as contrast exposure for the patients. Another important consideration is that the operators should be trained for the management of potentially life-threatening complications, like coronary perforation and cardiac tamponade.

\section{FUTURE DIRECTIONS}

The hybrid approach has been proven to be very effective and has also shown to reduce the rate of complications. The next step is to train potential CTO operators so that the correct diffusion of this algorithm will further improve success rates, especially in lower CTO PCI volume centers. In our view proctoring is a crucial step for a steep increase in expertise of new CTO operators. Moreover, we need efforts to further improve patient comfort and to reduce access related complications by minimizing the use of dual access, large bore catheters and femoral approach when it is possible, without impacting the efficiency of the hybrid algorithm.

\section{DECLARATIONS}

\section{Authors' contributions}

Made substantial contributions to conception and design of the study and performed data analysis and interpretation: Zivelonghi C, Budassi S, Agostoni P

Performed data acquisition, as well as provided administrative, technical, and material support: Budassi S, Zivelonghi C, Agostoni P

\section{Availability of data and materials}

Not applicable.

\section{Financial support and sponsorship}

None.

\section{Conflicts of interest}

All authors declared that there are no conflicts of interest.

\section{Ethical approval and consent to participate}

Not applicable.

\section{Consent for publication}

Not applicable.

\section{Copyright}

(c) The Author(s) 2019. 


\section{REFERENCES}

1 Colombo A, Mikhail GW, Michev I, Iakovou I, Airoldi F, et al. Treating chronic total occlusions using subintimal tracking and reentry: the STAR technique. Catheter Cardiovasc Interv 2005;64:407-11.

2 Carlino M, Godino C, Latib A, Moses JW, Colombo A. Subintimal tracking and re-entry technique with contrast guidance: a safer approach. Cath Cardiovasc Interv 2008;72:790-6.

3 Lombardi WL. Retrograde PCI: what will they think of next? J invasive Cardiol 2009;21:543.

4 Galassi AR, Tomasello SD, Costanzo L, Campisano MB, Barrano G, et al. Mini-STAR as Bail-Out Strategy for Percutaneous coronary intervention of chronic total occlusion. Cath Cardiovase Interv 2012;79:30-40.

5 Surmely JF, Tsuchinake E, Katoh O, Nishida Y, Nakayama M, et al. New concept for cto recanalization using controlled antegrade and retrograde subintimal tracking: The cart technique. J Invasive Cardiol 2006;8:334-8.

6 Brilakis ES, Grantham JA, Rinfret S, Wyman RM, Burke MN, et al. A percutaneous treatment algorithm for crossing coronary chronic total occlusions. JACC Cardiovasc Interv 2012;5:367-79.

7 Tajti P, Brilakis ES. Chronic Total Occlusion Percutaneous Coronary Intervention: Evidence and Controversies. J Am Heart Assoc 2018;12;7.

8 Harding SA, Wu EB, Lo S, Lim ST, Ge L, et al. A New Algorithm for Crossing Chronic Total Occlusions From the Asia Pacific Chronic Total Occlusion Club JACC Cardiovasc Interv 2017;10:2135-43.

9 Christopoulos G, Karmpaliotis D, Alaswad K, Yeh RW, Jaffer FA, et al. Application and outcomes of a hybrid approach to chronic total occlusion percutaneous coronary intervention in a contemporary multicenter US registry. Int J Cardiol 2015;198:222-8.

10 Maeremans J, Walsh S, Knaapen P, Spratt JC, Avran A, et al. The Hybrid Algrithm for Treating Chronic Total Occlusions In Europe. The RECHARGE Registry. J Am Coll Cardiol 2016;68:1958-70.

11 Bakker EJ, Maeremans J, Zivelonghi C, Faurie B, Avran A, et al. Fully Transradial Versus Transfemoral Approach for Percutaneous Intervention of Coronary Chronic Total Occlusions Applying the Hybrid Algorithm. Circ Cardiovasc Interv 2017;10:e005255.

12 Morino Y, Abe M, Morimoto T, Takeshi Kimura, Yasuhiko Hayashi, et al. Predicting Successful Guidewire Crossing Through Chronic Total Occlusion of Native Coronary Lesions Within 30 Minutes. JACC Cardiovascular Intervention 2011;4:213-21.

13 Werner G. The BridgePoint devices to facilitate recanalization of chronic total coronary occlusions through controlled subintimal reentry. Expert Rev Med Devices 2011;8:23-9.

14 Rinfret S, Joyal D, Nguyen CM, Bagur R, Hui W, et al. Retrograde recanalization of chronic total occlusions from the transradial approach; early Canadian experience. Catheter Cardiovasc Interv 2011;78:366-74.

15 Hamon M, Mehta S, Steg PG, Faxon D, Kerkar P, et al. Impact of transradial and transfemoral coronary interventions on bleeding and net adverse clinical events in acute coronary syndromes. EuroIntervention 2011;7:91-7.

16 Ferrante G, Rao SV, Juni P, Da Costa BR, Reimers B, et al. Radial Versus Femoral Access for Coronary Interventions Across the Entire Spectrum of Patients With Coronary Artery Disease: A Meta-Analysis of Randomized Trials. JACC Cardiovasc Interv 2016;9:1419-34.

17 Di Mario C, Ramasami N. Techniques to enhance guide catheter support. Catheter Cardiovasc Interv 2008;72:505-12.

18 Schumacher SP, Stuijfzand WJ, Opolski MP, van Rossuma AC, Nap A, et al. Percutaneous coronary intervention of chronic total occlusions: when and how to treat. Cardiovascular Revascularization Medicine 2018;8:897-988.

19 Galassi A, Grantham A, Kandzari D, Lombardi W, Moussa I, et al. Percutaneous Treatment of Coronary Chronic Total Occlusion Part 2: Technical Approach. Interv Cardiol 2014;9:201-7.

20 Michael TT, Papayannis AC, Benerjee S, Brilakis ES. Subintimal Dissection/Re-entry Strategies in Coronary Chronic Total Occlusion Interventions. Circ Cardiovasc Interv 2012;5:729-38.

21 Brilakis ES, Grantham JA, Thompson CA, DeMartini TJ, Prasad A, et al. The retrograde approach to coronary artery chronic total occlusions: a practical approach. Catheter Cardiovasc Interv 2012;79:3-19.

22 Tsuchikane E, Katoh O, Kimura M, Nasu K, Kinoshita Y, et al. The first clinical experience with a novel catheter for collateral channel tracking in retrograde approach for chronic coronary total occlusions. JACC Cardiovasc Interv 2010;3:165-71.

23 Brilakis ES. Manual of Coronary Chronic Occlusion Interventions. Elsevier 2014; doi: 10.1016/B978-0-12-420129-3.00019-2.

24 Zivelonghi C, van Kuijk JP, Suttorp MJ, van der Heyden JAS, Eefting FD, et al. Implementing a minimally invasive approach (combining radial approach, small guiding catheters and minimization of double access) for coronary chronic total occlusion intervention according to the hybrid algorithm: The Minimalistic Hybrid Algorithm. Int J Cardiol 2019;15;283:84-7. 